\title{
Reduction of the Pseudoinverse of a Hermitian Persymmetric Matrix
}

\author{
By Marvin J. Goldstein
}

\begin{abstract}
When the pseudoinverse of a Hermitian persymmetric matrix is computed, both computer time and storage can be reduced by taking advantage of the special structure of the matrix.
\end{abstract}

For any matrix $M$, let $M^{t}$ and $M^{*}$ denote its transpose and conjugate transpose, respectively. Let $J$ be a permutation matrix whose elements along the southwestnortheast diagonal are ones and whose remaining elements are zeros. Note that

$$
J=J^{*}=J^{-1} \text {. }
$$

Definition 1. $M$ is persymmetric if $J M^{*} J=\bar{M}$, the complex conjugate of $M$.

Note that all Toeplitz matrices $\left(t_{i j}=t_{i+1, j+1}\right)$ are persymmetric. $=-M$.

Definition 2. $M$ is centrosymmetric if $J M J=M$; skew-centrosymmetric if $J M J$

Note that if a persymmetric matrix is symmetric, it is centrosymmetric; if a persymmetric matrix is skew $\left(M^{t}=-M\right)$ it is skew-centrosymmetric. It is clear, therefore, that the real and imaginary parts of a Hermitian persymmetric matrix are centrosymmetric and skew-centrosymmetric, respectively.

In [2], matrix forms for the pseudoinverse of a centrosymmetric matrix are given in terms of the pseudoinverses of smaller matrices. Similar matrix forms for the pseudoinverse of a skew-centrosymmetric matrix are given in [3]. In this paper, we show that the pseudoinversion of a Hermitian persymmetric matrix reduces to the pseudoinversion of a real symmetric matrix of the same order.

Definition 3. The pseudoinverse $A^{+}$of any matrix $A$ is uniquely defined by the matrix equations:

$$
A A^{+} A=A, \quad A^{+} A A^{+}=A^{+}, \quad\left(A^{+} A\right)^{*}=A^{+} A, \quad\left(A A^{+}\right)^{*}=A A^{+} .
$$

It is straightforward to verify that

$$
\begin{array}{rlrl}
A^{+} & =A^{-1} & & (A \text { nonsingular }), \\
(U A V)^{+} & =V^{*} A^{+} U^{*} & & (U, V \text { unitary }), \\
\bar{A}^{+} & =\overline{A^{+}} & & \\
\left(A^{*}\right)^{+} & =\left(A^{+}\right)^{*}, &
\end{array}
$$

Received August 13, 1973.

AMS (MOS) subject classifications (1970). Primary 15A09, 15A57, 65F20.

Key words and phrases. Persymmetric, Hermitian, centrosymmetric, skew-centrosymmetric, pseudoinverse, Toeplitz. 
and

$$
\begin{array}{rlrl}
D^{+} & =\operatorname{diag}\left(d_{1}, \ldots, d_{n}\right) \quad(D \text { diagonal }), \\
d_{i} & =1 / D_{i i} & & \text { if } D_{i i} \neq 0, \\
& =0 & & \text { otherwise },
\end{array}
$$

satisfy (1).

If $P$ is an even order Hermitian persymmetric matrix that is split into real and imaginary parts, it may be partitioned as

$$
P=\left(\begin{array}{cc}
K & H J \\
J H & J K J
\end{array}\right)+i\left(\begin{array}{cc}
S & N J \\
-J N & -J S J
\end{array}\right),
$$

where $K, H, N$ are real and symmetric, and $S$ is real and skew. (Note that any complex centrosymmetric (skew-centrosymmetric) matrix of even order can be written in the partitioned form of the real (imaginary) part in (7) with $K$ and $H$ ( $S$ and $N$ ) complex.)

The pseudoinverse of $P$ may be partitioned in the same form:

$$
P^{+}=\left(\begin{array}{cc}
B & C J \\
J C & J B J
\end{array}\right)+i\left(\begin{array}{cc}
F & G J \\
-J G & -J F J
\end{array}\right),
$$

because it is also Hermitian persymmetric by (5), (3), (4) and Definition 1. The form of (7) suggests applying $P$ to matrices of special form.

Let $U, V$ be real matrices conformable with $K$ such that

is nonsingular. Then, by [1],

$$
T=\left(\begin{array}{c}
U \\
J U
\end{array}\right)+i\left(\begin{array}{c}
V \\
-J V
\end{array}\right)
$$

$$
P T=T \Lambda \quad(\Lambda \text { diagonal })
$$

if and only if

$$
Q \tilde{T}=\tilde{T} \Lambda
$$

where

$$
Q=\left(\begin{array}{cc}
K+H & -(S-N) \\
S+N & K-H
\end{array}\right), \quad \tilde{T}=\left(\begin{array}{l}
U \\
V
\end{array}\right)
$$

Note that $Q$ is real and symmetric.

Now, suppose $\tilde{T}$ is orthogonal and satisfies (9). Then $T^{*} T=T T^{*}=2 I$. Thus, $P=0.5 T \Lambda T^{*}$ and, by direct substitution into (1),

$$
P^{+}=0.5 T \Lambda^{+} T^{*}, \quad Q^{+}=\tilde{T} \Lambda^{+} \tilde{T}^{*} .
$$

Hence, with $P^{+}$defined by (8), $P^{+} T=T \Lambda^{+}$and, by [1],

$$
Q_{1} \tilde{T}=\tilde{T} \Lambda^{+},
$$

where 


$$
Q_{1}=\left(\begin{array}{cc}
B+C & -(F-G) \\
F+G & B-C
\end{array}\right)
$$

But $Q_{1}=Q^{+}$by (11) and (12).

Thus, $P^{+}$can be obtained by computing $B, C, F$, and $G$ from $Q^{+}$with a reduction in both storage and computer time.

If $P$ is real and symmetric, then

$$
N=S=0
$$

and

$$
Q^{+}=\operatorname{diag}\left((K+H)^{+},(K-H)^{+}\right)=\operatorname{diag}(B+C, B-C) .
$$

In [2], the pseudoinversion of an arbitrary even order centrosymmetric matrix in the partitioned form of the real part of (7) is reduced also to the pseudoinversion of the matrices $K+H$ and $K-H$.

If $P$ is pure imaginary, then

$$
K=H=0
$$

and

$$
Q^{+}=\left(\begin{array}{cc}
0 & (S+N)^{+} \\
-(S-N)^{+} & 0
\end{array}\right)=\left(\begin{array}{cc}
0 & -(F-G) \\
F+G & 0
\end{array}\right) .
$$

In [3], the pseudoinversion of an arbitrary even order skew-centrosymmetric matrix in the partitioned form of the imaginary part of (7) is reduced also to the pseudoinversion of the matrices $S+N$ and $S-N$.

When the order of $P$ is odd, the analogous forms are

$$
\begin{aligned}
P & =\left(\begin{array}{ccc}
K & c & H J \\
c^{t} & \rho & c^{t} J \\
J H & J c & J K J
\end{array}\right)+i\left(\begin{array}{ccc}
S & d & N J \\
-d^{t} & 0 & d^{t} J \\
-J N & -J d & -J S J
\end{array}\right), \\
Q & =\left(\begin{array}{ccc}
K+H & \sigma c & -S+N \\
\sigma c^{t} & \rho & \sigma d^{t} \\
S+N & \sigma d & K-H
\end{array}\right) \quad(\sigma=\sqrt{ } 2),
\end{aligned}
$$

where $c, d$ are real column vectors conformable with $J$.

Acknowledgment. The author is grateful to the referee for comments improving this paper.

Naval Underwater Systems Center

New London Laboratory

New London, Connecticut 06320

1. M. J. Goldstern, "Reduction of the eigenproblem for Hermitian persymmetric matrices," Math. Comp., v. 28, 1974, pp. 237-238.

2. W. C. Pye, T. L. Boullion \& T. A. Atchison, "The pseudoinverse of a centrosymmetric matrix," Linear Algebra and Appl., v. 6, 1973, pp. 201-204.

3. G. L. Shurbet, T. O. Lewis \& T. L. Boullion, "Generalized inverses of skew-centrosymmetric matrices," Math. Comp. (To appear.) 